$\Delta$ Palabras clave/ Extensión universitaria proyecto piloto, equipo comunitario, residuos urbanos.

$\Delta$ Keywords/ University outreach, pilot project, community team, urban waste.

$\Delta$ Recepción/ 17 octubre 2017

$\Delta$ Aceptación/ 14 febrero 2018

\title{
Una experiencia de extensión universitaria: Proyecto para cooperativa de reciclaje de residuos sólidos.
}

\section{An experience with University outreach: \\ A Project for a Solid Waste Recycling \\ Cooperative.}

\section{Renata Pereira}

Arquitecta, Universidad Federal de Minas Gerais, Brasil.

Magister en Urbanismo, Pontificia Universidad Católica de Campinas, Brasil.

Doctora, Programa de Posgrado de la Facultad de Arquitectura y Urbanismo, Universidad São Paulo, Brasil.

Profesora, Facultad de Arquitectura y Urbanismo: Profesora e Investigadora, Programa de Posgrado en Arquitectura y Urbanismo, Pontificia Universidad Católica de Campinas, Brasil. renata.baesso@puc-campínas.edu.br

\section{Vera Luz}

Arquitecta, Universidad Mackenzie, Brasil. Doctora, Programa de Posgrado de la Facultad de Arquitectura y Urbanismo, Universidad São Paulo, Brasil.

Profesora, Facultad de Arquitectura y Urbanismo; Profesora e Investigadora, Programa de Posgrado en Arquitectura y Urbanismo, Pontificia Universidad Católica de Campinas, Brasil. Oradora Invitada, Fundación Vanzolini, Processo AQUA-HQE, São Paulo, Brasil.

veraluz@puc-campinas.edu.br

\section{Daniel Ribeiro}

Arquitecto, Pontificia Universidad Católica de Campinas, Brasil.

Magíster en Urbanismo, Pontificia

Universidad Católica de Campinas, Brasil. dhrmuz@gmaill.com

RESUMEN/ Este trabajo presenta la experiencia del Programa de Educación Tutorial (PET) del Ministerio de Educación, impartido a nivel de enseñanza de graduación, en la Facultad de Arquitectura y Urbanismo de la Pontificia Universidad Católica (PUC) de Campinas, con la participación de once alumnos bajo la tutoría de una docente, y coordinación de la actividad realizada por dos profesoras. El objetivo del programa fue desarollar un proyecto piloto central de selección de residuos, atendiendo a la solicitud de una cooperativa de reciclaje. La metodología aplicada involucró enseñanza, investigación y extensión universitaria, participación de la comunidad, el poder público y el patrocinador. Se pretendió establecer paradigmas para que el trabajo pudiera ser el más cercano a la realidad desde el punto de vista de la conceptualización, actividades prácticas, interfaz comunitaria y etapas de desarrollo. Se constituyó un proyecto atendiendo a las demandas del programa, especificaciones funcionales y de manejo, cuya morfología y aspectos constructivos ejemplifiquen los aspectos sustentables del tema. ABSTRACT/ This work introduces the experiences of the Tutorial Education Program (PET) of the Ministry of Education, delivered at the graduate level for the School of Architecture and Urban Development of the Pontificia Universidad Católica (PUC) de Campinas. The program engaged eleven students with the guidance of a professor and activities coordinated by two professors. The purpose of the program was to develop a central waste sorting pilot project in response to the requirements by a recycling cooperative. The methodology used involved university teaching, research and outreach; and participation by the community, the public and the sponsor. Paradigms were expected to be created in order to bring the work closer to reality from the standpoint of its conceptualization, practical activities, community interface and development stages. A project was established based on the needs of the program and on functional and management specifications, the morphology and construction aspects of which can be an example of the sustainable aspects of the topic.

\section{INTRODUCCIÓN.}

discute el método de desarrollo del proyecto arquitectónico de la centra de seleción de residuos sólidos de una cooperativa ubicada en el municipio de Valinhos, Región Metropolitana de Campinas-SP, Brasil.

La oportunidad concreta de elaborar un proyecto involucrando actores diversos -la universidad, organizaciones no gubernamentales (ONGs), el poder público municipal y la iniciativa privada-, permitió la construcción de un método que alinea experiencias guiadas por la indisociabilidad entre enseñanza, investigación y extensión, característica del Programa de Educación Tutorial (PET). El programa estuvo constituido por alumnos de pregrado, con tutoría de un docente, dedicados al desarrollo de actividades que contribuyeron al incremento de habilidades en su formación desde el primer año de la graduación hasta la conclusión del curso. En Brasil, el Programa PET fue creado en 1979 en conjunto con las iniciativas de fortalecimiento de la enseñanza superior conducidas por la Coordinación de Perfeccionamiento de Personal de Nivel Superior (CAPES). Su gestión fue asumida por la Secretaría de Educación Superior del Ministerio de la Educación (SESU) MEC) en 1999. Actualmente cuenta con 842 grupos, ligados a las diversas áreas del conocimiento, en 121 instituciones públicas, privadas y comunitarias de enseñanza superior. La constitución de proyectos y acciones se fundamenta en el trabajo colectivo y colaborativo como herramienta. Dadas sus características -participación voluntaria, objetivos comunes, libertad de expresión, disolución de jerarquía- se constituye como una herramienta para el perfeccionamiento académico de todos los involucrados: alumnos, tutores, profesores colaboradores y comunidad estudiantil (Ferreira, Martins y Freitas 2007). 


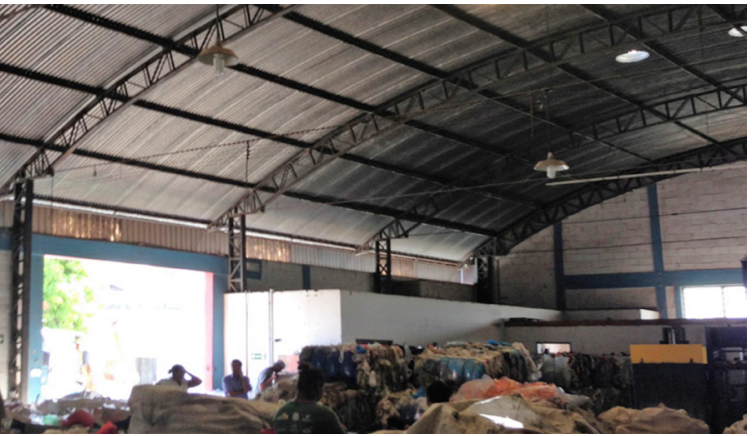

Imagen 1. Cooperativa de Seleción de Residuos Sólidos (RECOOPERA) en la actualidad (fuente: Renata Baesso).

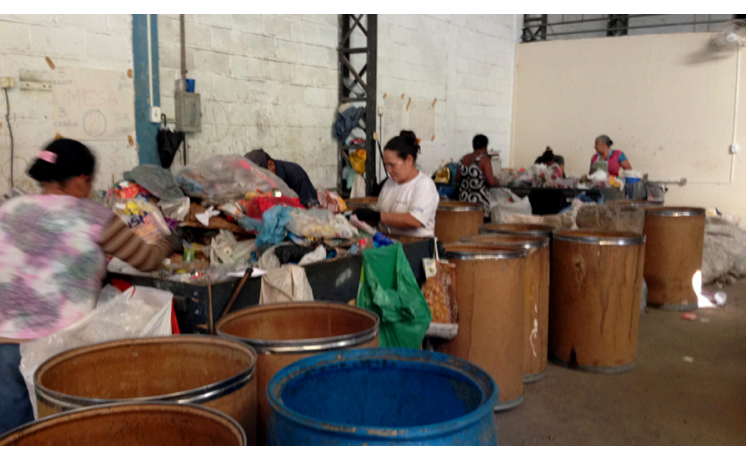
Imagen 2. Cooperativa de Seleción de Residuos Sólidos
(RECOOPERA) en la actualidad (fuente: Renata Baesso).

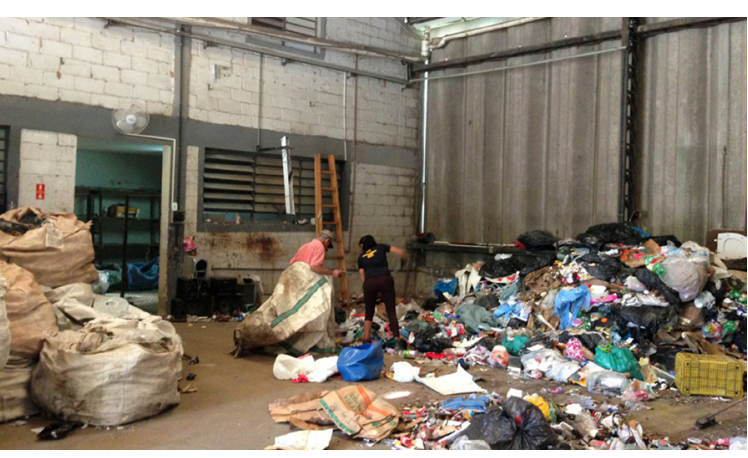

Imagen 3.

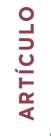

온
Creado en 1992, el PET Arquitectura y Urbanismo de la PUC de Campinas se compone de un profesor tutor, doce alumnos becarios y hasta seis alumnos voluntarios, quienes se implican en el programa de la misma forma que los becarios. El grupo cuenta con un taller de proyecto propio para el desarrollo de investigaciones, proyectos y estudios. Dado que uno de los objetivos del programa es que los becarios sean agentes multiplicadores de conocimiento, otros alumnos del curso también frecuentan el taller del PET y participan de aciones en grupos liderados por los becarios.

Profesores integrantes de la Facultad de Arquitectura pueden ser invitados a integrar equipos para el desarrollo de determinados trabajos o proyectos, de acuerdo con sus especialidades y compromisos temáticos, potenciando así el intercambio de conocimiento y experiencia

Desde su origen, el PET ha contribuido a la discusión del Proyecto Pedagógico del Curso de Arquitectura y Urbanismo, que se propone abordar de forma reflexiva y crítica las temáticas relativas a la cuestión del territorio, la ciudad y la vivienda, estableciendo interfaces entre las actividades de enseñanza, investigación y extensión, por medio de las cuales se busca estrechar lazos con la comunidad. En consonancia con la tradición humanista, que caracteriza la actividad de la arquitectura y el urbanismo, y con la misión de esta universidad-confesional, filantrópica y comunitaria-, las acciones pedagógicas se orientan hacia el desarrollo de conductas y actitudes que tengan como fundamento:

- La calidad de vida de los habitantes de los asentamientos humanos y la calidad material del medio ambiente construido y su durabilidad.

- El uso de las tecnologías en relación a las necesidades sociales, culturales, estéticas y económicas de las comunidades.

- El equilibrio ecológico y el desarrollo sostenible del entorno natural y construido.

- La valorización y la preservación de la arquitectura, del urbanismo y del paisaje como patrimonio y de responsabilidad colectiva.
A principios del año 2013, el PET construyó una asociación con la Cooperativa de Seleción de Residuos Sólidos (RECOOPERA), que tuvo por objetivo el desarrollo del proyecto arquitectónico de su central de selección, el cual se construiría en un terreno facilitado por el Ayuntamiento Municipal de Valinhos, con recursos de la empresa privada Ruta de las Banderas. La RECOOPERA solicitó la realización del proyecto del nuevo edificio, que vendría a sustituir a su actual central de selección, la cual funciona en una nave provisional adaptada de modo precario, que fue cedida por el Ayuntamiento Municipal. El PET Arquitectura y Urbanismo consideró el convenio del proyecto como una oportunidad de trabajar, de forma interdisciplinaria, un proyecto que uniera la investigación a la práctica del proyecto, en respuesta a necesidades reales de una comunidad con múltiples carencias. La RECOOPERA cuenta con 40 miembros y procesa cerca de 130 toneladas de residuos por mes; integra el Centro de Referencia en Cooperativismo y Asociativismo (CRCA), que tiene por objetivo rescatar a personas en situación de vulnerabilidad económica y social que, organizadas en cooperativas populares de colecta, separación y venta de material reciclable legalmente constituidas, son capaces de provocar transformaciones positivas en su medio. Apoyado en los preceptos de la economía solidaria, el CRCA asesora a cooperativas en las que ciudadanos alejados del mercado de trabajo encuentran la reinserción social, al mismo tiempo que desarrollan actividades que constituyen un aporte en la recuperación del medio ambiente. Parte de ellas están asociadas también a la Central Solidaria de Ventas (RECICLAMP), que tiene como objetivo ampliar las ventas de materiales reciclables a las empresas recicladoras, sin intermediarios.

Los proyectos desarrollados por las dos ONG buscan alianzas con diferentes sectores de la sociedad, estimulando a cada cooperativa a alcanzar una gestión autosustentable, alejándose de acciones meramente asistenciales? 
La correcta destinación de los residuos sólidos es una condición primordial para una ciudad sustentable, ya que cuando se manejan adecuadamente, adquieren valor comercial y pueden ser reciclados, constituyendo nuevas materias primas o nuevos insumos. En palabras de Silva (2017): La actividad de reciclaje se ha estructurado como un sector económico de relativa importancia a medida que la cuestión de los residuos sólidos se ha convertido en los últimos años en uno de los problemas centrales en términos de planificación urbana y gestión pública en prácticamente todas las grandes ciudades del mundo. Esta formada por un conjunto de operaciones interconectadas cuya finalidad es la reintroducción de los materiales reciclables en los procesos de producción para ser transformados nuevamente en insumos productivos. En este sector de actividades, tiene un papel fundamental la figura de los recolectores de materiales reciclables, que suministran los insumos básicos para la etapa de beneficio y transformación de los materiales con la finalidad de reutilización en el proceso productivo (2017: 7).

En Brasil, la aprobación de la Política Nacional de Residuos Sólidos (PNRS) en 2010, trajo instrumentos para que los municipios vinieran a responsabilizarse por la gestión, buscando la superación de problemas ambientales, sociales y económicos derivados del manejo inadecuado de los residuos sólidos, y está basada en el concepto de responsabilidad compartida, donde las industrias, comerciantes, ayuntamientos y consumidores son co-responsables por la correcta destinación de los residuos sólidos. El trabajo de los recolectores de materiales reutilizables y reciclables, reconocido por el Ministerio de Trabajo y Empleo desde 2002, fue incluido en la implementación de la PNRS, pues estos actúan en las actividades de recolección selectiva, clasificación procesamiento y comercialización de los residuos, contribuyendo de forma significativa a la cadena productiva del reciclaje.

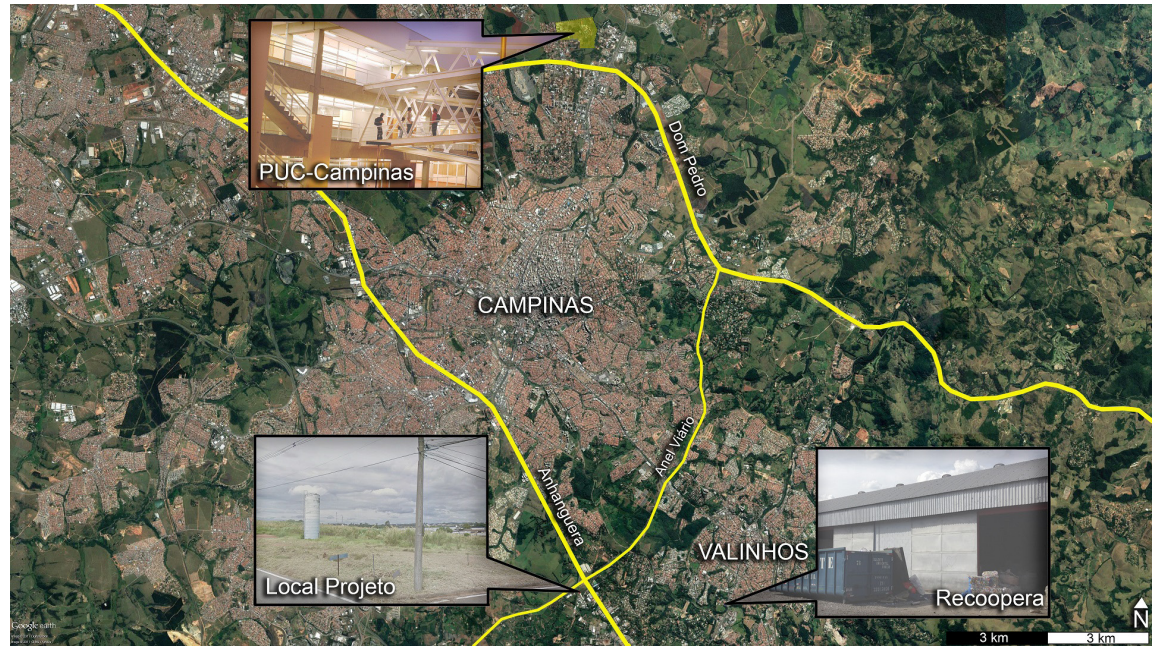

Figura 1. Situación Regional (fuente: Los autores en base a Googlemaps).

Históricamente, la actuación de los recolectores se realiza bajo condiciones muy precarias de trabajo, de forma autónoma e informal, dispersa por las calles y en los depósitos de basura. Así, en palabras de Silva (2017):

Para enfrentar esta dura realidad de pobreza y exclusión social, un contingente considerable de recolectores -trabajadores involucrados en diferentes eslabones de la cadena del reciclaje- han buscado organizarse bajo diferentes estrategias de acción colectiva. Esta organización puede ser observada tanto en términos de representación política, con la movilización de los trabajadores y sus familiares en torno a una categoría profesional en busca de reconocimiento social y derechos de ciudadanía, en términos económicoproductivos, con la formación de asociaciones y/o cooperativas con el fin de sumar fuerzas a través del trabajo colectivo (Silva 2017: 8).

Para alcanzar buenos niveles de eficiencia, los emprendimientos de recolectores necesitan infraestructura y equipos fundamentales para el ejercicio de su actividad, tales como: sede propia, nave con espacio adecuado para la clasificación y almacenamiento de residuos, vehículos (preferentemente camiones para la recolección selectiva y transporte de los residuos), teléfonos, ordenadores con acceso a internet, mesas y bolsas para seleccionar los residuos, prensas para comprimir y carritos manuales para transporte, entre otros (Pepinelli 2011 en Silva 2017: 32).

Tradicionalmente, las Universidades brasileñas han tendido a priorizar las demandas del mercado y concentrar sus estrategias de formación atendiendo a los futuros empleadores de sus estudiantes (Singer 2008). El proyecto en cuestión atiende a una parte de la población de la RMC históricamente excluida y no inserta en el mercado de trabajo tradicional. El desarrollo del proyecto arquitectónico de la nueva sede de la Cooperativa es una oportunidad para que los estudiantes apoyen un emprendimiento de economía solidaria, involucrándose con el trabajo de organizaciones no gubernamentales. La asociación entre academia, ONGs, poder público municipal e iniciativa privada en una experiencia concreta, permite a los alumnos desarrollar metodologías de trabajo concernientes a la teoría y la práctica, y que retroalimentan los contenidos de las disciplinas y proyectos de investigación produciendo nuevos saberes que pueden ser puestos al servicio de la comunidad. 


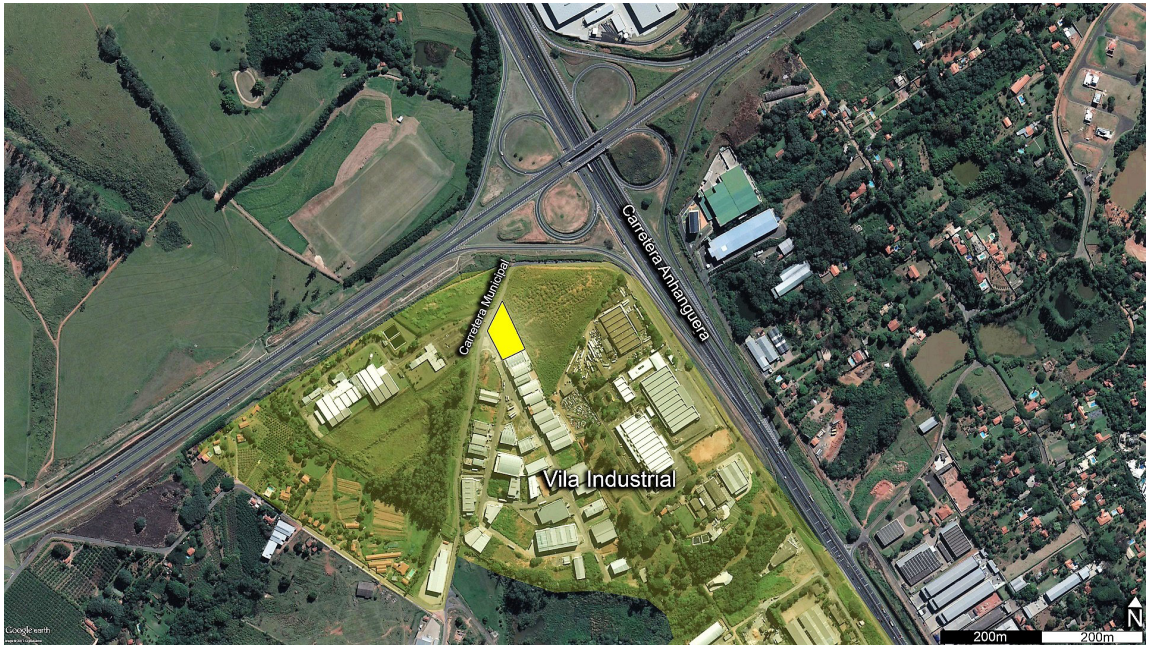

Figura 2. Ubicación del proyecto (fuente: Los autores en base a Googlemaps).

A partir de la discusión de la metodología empleada, se busca reflexionar sobre el potencial de convenios como este en la elaboración de prácticas de investigación y enseñanza del proyecto de arquitectura en interfaz con demandas reales y participación social, tratándose esta vez de un proceso piloto en el ámbito de la arquitectura, y con proyección de aplicarse de manera ampliada, dada la escala de los desafíos

METODOLOGÍA DE DESAROLLO DEL PROYECTO. Perspectivas Generales.

Se trataba de realizar un provecto piloto

de arquitectura comprendiendo de manera articulada los aspectos de enseñanza, investigación y extensión universitaria, con un conjunto de once estudiantes, una profesora tutora y una profesora coordinadora invitada, involucrando al equipo en todo el proceso de una experiencia que solo sería efectiva en la medida en que se atendiesen las demandas

medio de su representante, que fue en esta ocacasión un científico social comprometido con la misma: aspectos técnicos y de la maquinaria involucrada, sistemas de manejo, programa, flujos y operación, demandas comunitarias, compromisos educativos ambientales, adecuación, salubridad y comodidad de los espacios, potencialidades del terreno, legislación relativa, coherencia estructural y constructiva, y costes compatibles para la viabilidad de ejecución de acuerdo con los límites de los patrocinadores (empresa privada con apoyo institucional del ayuntamiento local). Se pretendió constituir una morfología cuyo reciclaje de residuos urbanos, dando fuerza simbólica al programa e imprimiendo una

\section{tipología replicable como sistema.}

\section{Participación con la Comunidad}

Cooperada. El Coordinador de la CRCA.

José Ronaldo Salles Fernandes y la

Presidenta de la Recoopera, Janine Silva Azevedo, intermediaron la participación comunitaria con los tutores y alumnos, en reuniones en el actual galpón de procesamiento de residuos, donde fue posible enterarse de los procesos productivos, del trabajo de inclusión social, de los valores generados y de la renta, de los aspectos precarios existentes y de las expectativas en relación al nuevo edificio. ejemplo pudiera evocar el propio tema del
La manifestación sobre la importancia de la convivencia y de la responsablidad educativa asumida por la cooperativa con respecto a la demanda de espacios para la recepción de estudiantes y visitantes, fue

\section{notable y conmovedora.}

\section{Enseñanza como ensayo de una} experiencia realizable y aproximación profesional. Para que el desarrollo de

proyecto se realizara de modo análogo al profesional como una experiencia preparatoria para los estudiantes, todas sus fases fueron discutidas y decididas en equipo y sus actividades fueron compartidas.

Se inició con la discusión del alcance general del proyecto, sus finalidades, plazos, competencias de las personas externas involucradas y del conjunto de alumnos, tutoría, coordinación y orientación. Asimismo, se discutió sobre la comunidad de la cooperativa de reciclaje, sobre quienes apoyaban el proyecto y sus patrocinantes. Se pasó al entendimiento del tema y sus implicancias, lo que denominamos 'identificación'. La palabra identificación abarca algo más profundo que el levantamiento de datos de programación dimensional o técnica, pues presupone la identidad con los mismos, sean estos materiales, humanos o ambientales. 


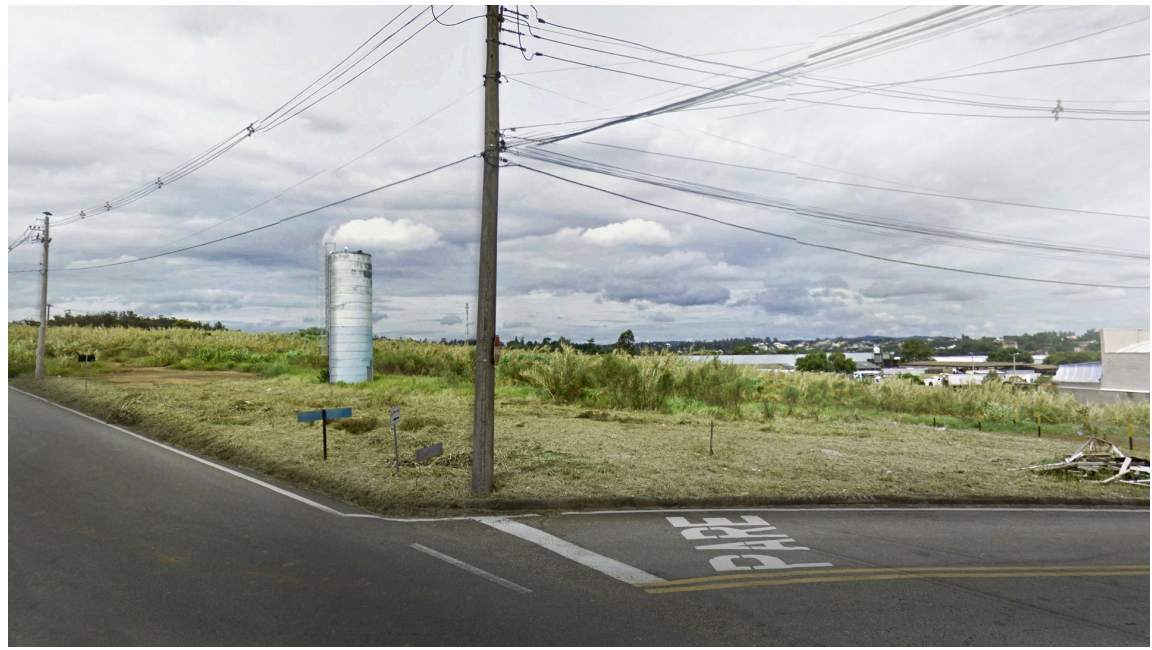

Imagen 4. Terreno del proyecto (fuente: Renata Baesso).

La investigación abarcó también, subgrupos de alumnos y el contacto con cooperativas

\section{semejantes:}

\section{- Maquinaria y equipo: cintas}

transportadoras y embudos; interfaz camiones/recepción de materiales; básculas de pesaje de carga sobre vehículos; básculas de material; prensas; empaquetamiento de los materiales; ascensores, plataformas elevadoras

y montacargas.

- Materiales y técnicas: especificaciones de paneles y tejas producidos a partir de restos de tubo de pasta de dientes picotados y prensados tipo ecoto $p^{\circledR}$; especificaciones de los paneles de residuos de envases de leche tetrapak; paneles ligeros de piso y cierres de madera laminada revestida con placa cementosa tipo wa/l ${ }^{\circledR}$

- Territorio: levantamiento planialtimétrico del lote; orientación solar y vientos; perímetro y área; declividades, accesos, relación con el entorno inmediato y con la región; vegetación; elementos preexistentes; legislación y parámetros urbanísticos con respecto al uso, coeficiente de aprovechamiento, tasa de ocupación, tasa de permeabilidad, plantillas de altura, retrocesos con las divisas, accesibilidad universal.
- Cooperativa de reciclaje: necesidades, especificaciones programáticas; hábitos, anhelos, expectativas en cuanto a los ambientes de trabajo, permanencia, higiene, guardia de pertenencias, descanso y recreo, alimentación, administración, reuniones, actividades educativas socioambientales; cantidad de integrantes, género y edad; proceso de producción, recepción de materiales, paquetes producidos, formas de clasificación, separación, almacenamiento, descarte; flujos de peatones y vehículos.

- Edificios análogos: morfologías, implantaciones, sistemas estructurales y constructivos, sistemas de operación, maquinaria y equipajes, programas, formas de uso y apropiación, organización del mobiliario

\section{- Entidades y personas apoyadoras y}

patrocinadores: área máxima posible de

patrocinio en la relación con costo de la edificación $/ \mathrm{m}^{2}$.

Los resultados de las investigaciones fueron presentados, comentados y discutidos con el objetivo de establecer en consenso lo siguiente:

- Programa de proyecto, definiendo ambientes y sus respectivas áreas, área total, organigrama funcional.
- Elección de la maquinaria y opciones de procesos de operación.

- Entendimiento del terreno, adecuación de alzados para orientación, porción edificante, aprovechamiento de los declives ante la organización de los espacios y operaciones; organización de flujos y permanencia de personas y vehículos.

A continuación, se investigaron posibilidades de implantación y volumetría (estudios de masas y de organización de los ambientes). Todos los alumnos fueron parte de experiencias individuales o en subgrupos, y se estableció paulatinamente, mediante la orientación comentada de las razones de las diversas elecciones, un proyecto de partida. Se concluyó que la mejor organización era el establecimiento de los sectores productivos en una planta baja con desniveles,

correspondientes al ciclo del material desde la recepción hasta el despacho, maquinaria implicada, accesos y flujos de personas y vehículos; donde también se ubicarían vestidores y servicios higiénicos, descanso y recreo, un pavimento superior parcial y un entresuelo con visión panorámica de los ambientes de trabajo, donde se ubicarían los sectores administrativos, de alimentación y multiuso, espacios para reuniones, permanencia y actividades educativas 


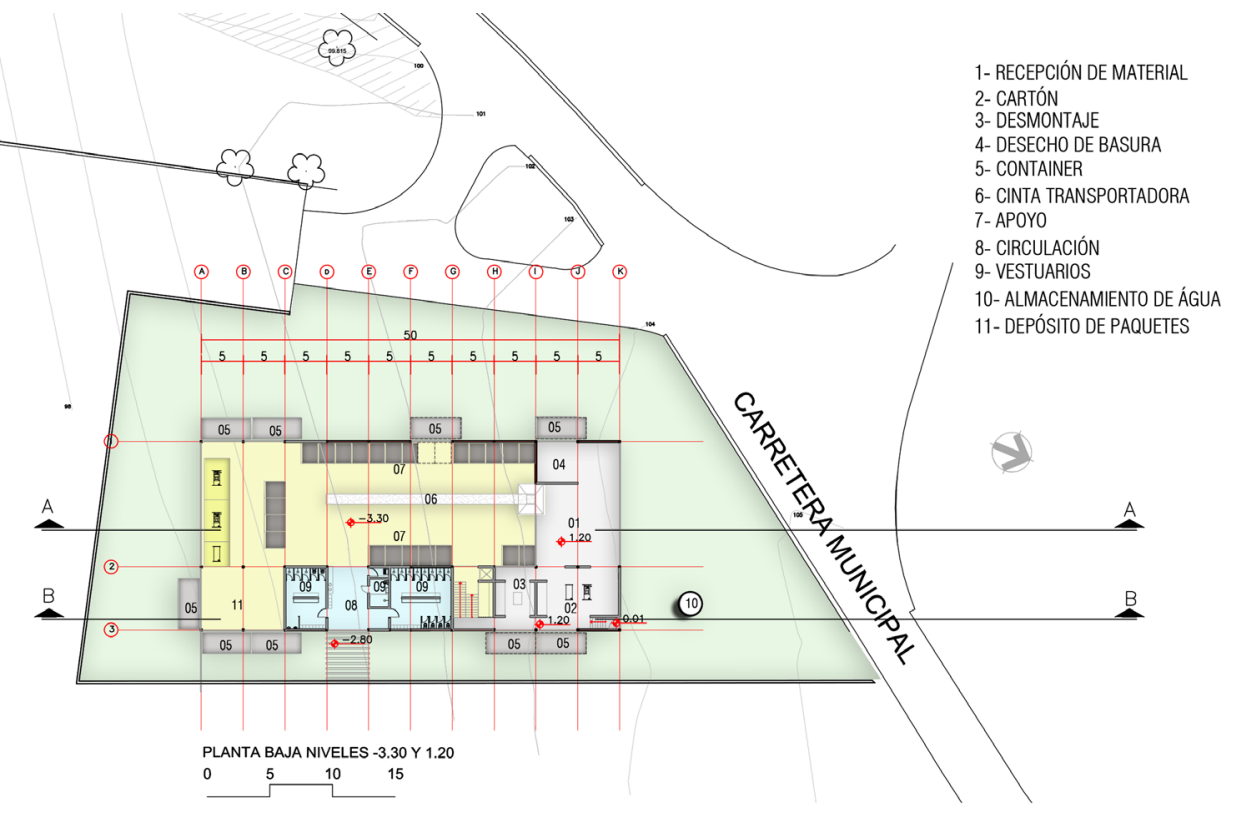

AREA DEL TERRENO $=3.548 .68 \mathrm{~m} 2$
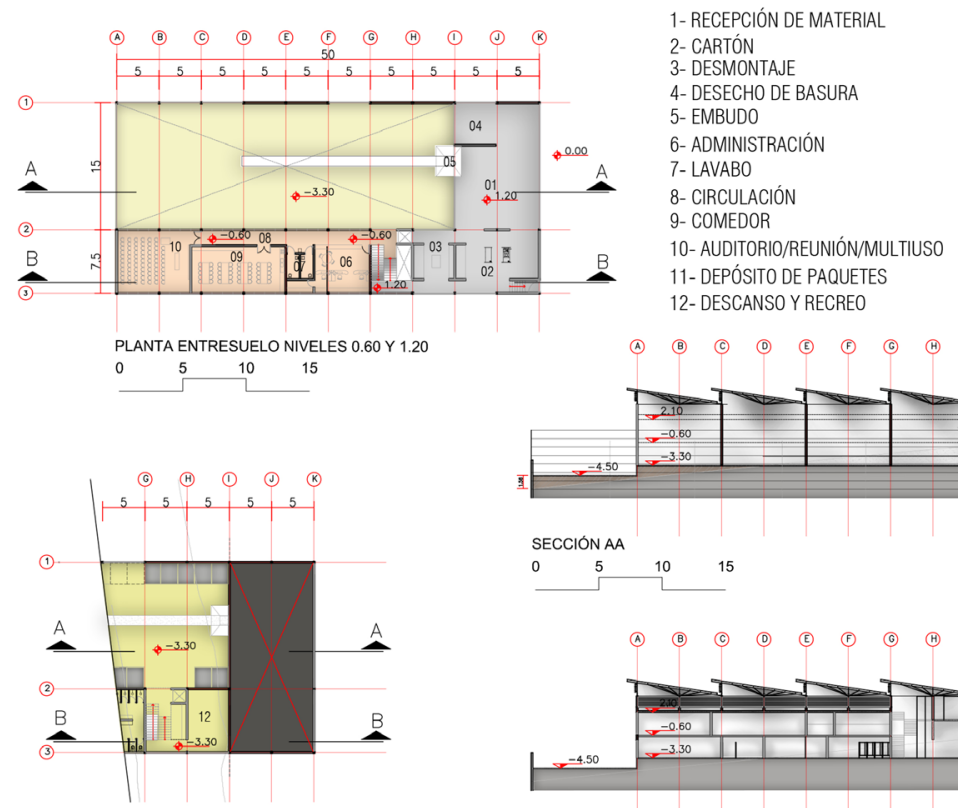

PLANTA BAJA NIVEL 3.30 SOB NIVEL 1.20

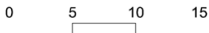

Figuras 3 y 4. Planta baja. Plantas bajo nivel $2.10 \mathrm{~m}$ y entresuelo. Secciones (fuente: Equipo de/ PET). socioambientales donde pudieran llevar a cabo encuentros que involucrasen a la cooperativa y a otras personas o entidades institucionales, educativas, de investigación y comunitarias. Se optó por juntar el sector administrativo con el de multiuso, evitando una jerarquía demasiado estratificada. Bajo la sección de desmontaje se incluyó una sala de descanso y recreo para los miembros de la cooperativa, articulada con los niveles del terreno.

En la fase siguiente, se estableció con precisión el tamaño, otorgando las dimensiones de $50 \mathrm{~m} \times 22,5 \mathrm{~m}$ al volumen construido a partir de un sistema modular articulador del paso estructural con la coordinación dimensional de los ambientes y la submodulación de los sistemas constructivos de cierres y aberturas. Se ajustaron los niveles con una exactitud relacionada a la maquinaria, sectores y ritmo de las escaleras, compatible con las proporciones de corte y relleno. El módulo estructural establecido fue de $5 \mathrm{~m}$ en 11 ejes en el sentido longitudinal y, en el sentido transversal, el vano de $15 \mathrm{~m}$ para la nave de trabajo y el de $7,5 \mathrm{~m}$ para el sector con doble pavimento - planta baja y entresuelo- contribuyendo con los sectores más compartimentados. Se optó por un volumen longitudinal, acompañando el sentido del terreno y, dada la anchura del edificio, un sistema de cobertura en sheds orientados hacia el sur, con iluminación y ventilación homogénea en todo el espacio. Para la parte de dos pavimentos, se eligió realizarla como un bloque, con estructura metálica para apoyo del piso, elevado de paneles ligeros tipo wal/巴 cubierta de losa plana y techo jardín accesible, optimizando la ventilación cruzada y la comodidad ambiental del entresuelo, además de ser posible la amortiguación de las aguas Iluvia e irrigación de pequeña huerta de hierbas aromáticas. Las aguas Iluvia de los sheds serían, en una segunda fase, recogidas en una cisterna para su reutilización. Existía una caja de agua preexistente -volumen cilíndrico vertical de origen industrial-, que fue aprovechada en la nueva edificación. El recorrido de los vehículos de carga se organizó en forma de anillo alrededor del edificio, y el acceso de peatones por la fachada oeste, ajustándose rampas y niveles de acceso de acuerdo a la inclinación del terreno. 

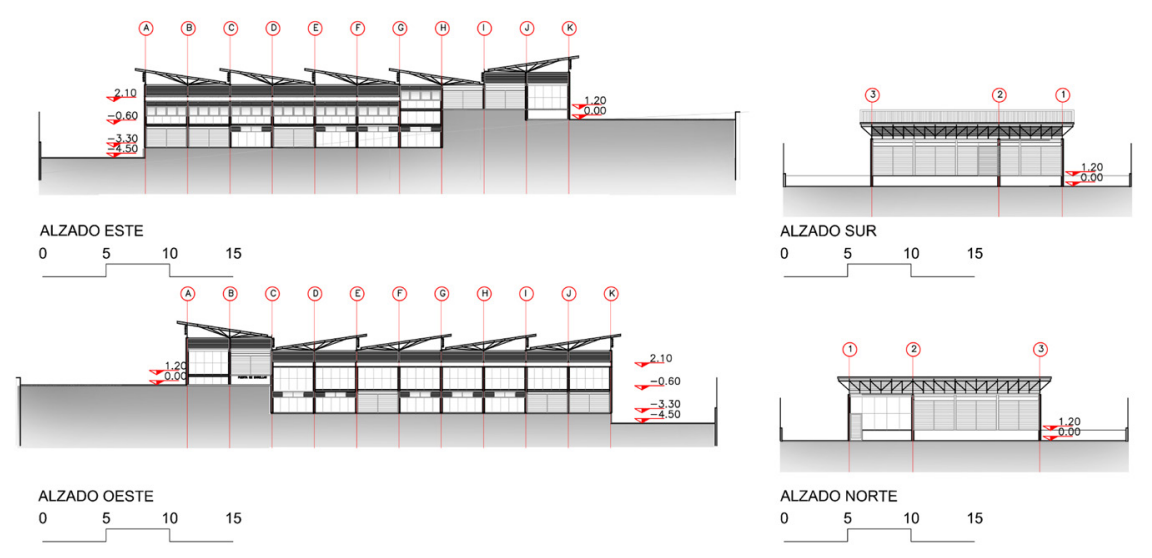

Figura 5. Elevaciones del proyecto (fuente: Equipo del PET).

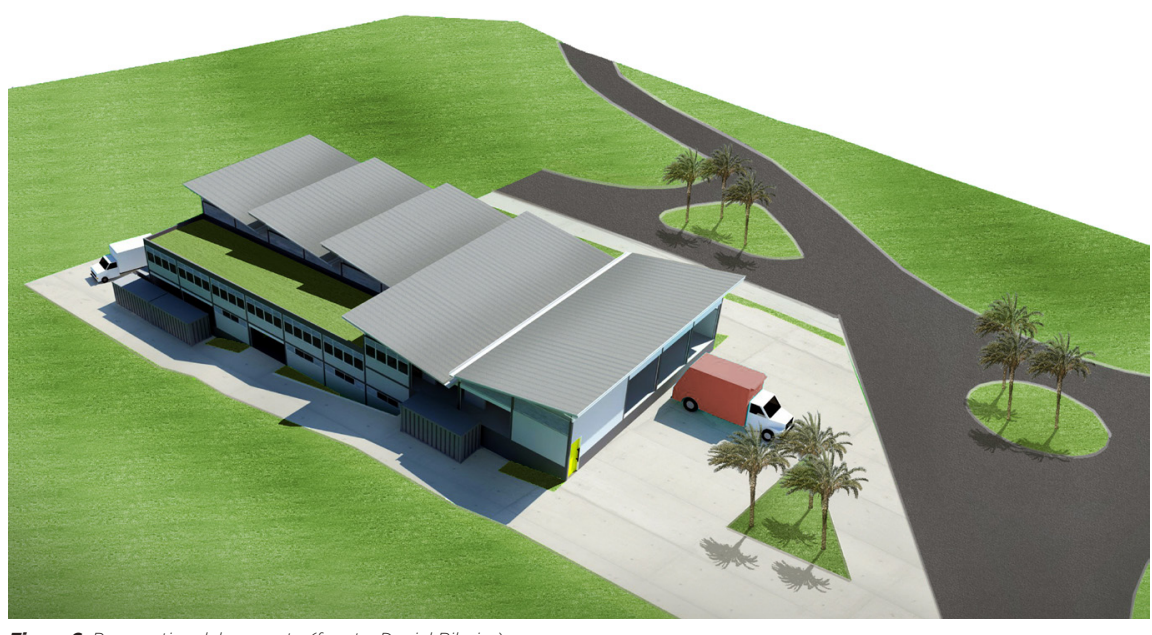

Figura 6. Perspectiva del proyecto (fuente: Danie/ Ribeiro).

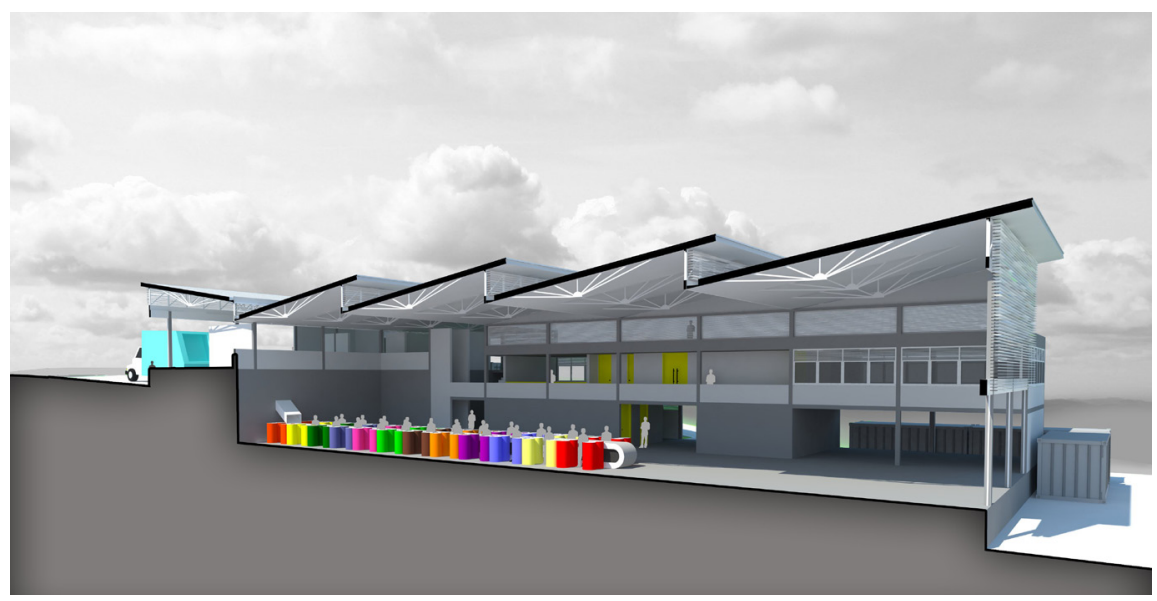

Figura 7. Perspectiva interior (fuente: Danie/ Ribeiro).
Las investigaciones de cada ambiente fueron realizadas por subgrupos para un correcto subdimensionamiento: vestidores, sala multiuso, cada sector productivo y demás sectores. Las opciones de organización del mobiliario, permanencia, operación y flujo se probaron y definieron. A continuación, se estudió la secuencia de cierres y aberturas mediante modulación establecida, utilizando marcos simples de acero y vidrio, puertas de enrollar de patrón industrial y algunas protecciones de celosías de acuerdo con la orientación solar.

El sistema estructural fue establecido por pilares y vigas de hormigón, la estructura de la cubierta en perfilados metálicos de dimensión reducida, en forma de vigas con piezas de travesaño. Las tejas, los cierres, las placas de celosias y las separaciones de los baños se realizaron todos en placas tipo ecotop ${ }^{\circledR}$. En los cierres externos, se superpuso una capa de aislante térmico y placas de envases tipo tetrapak, ambas de material reciclado. Todas las tuberías y componentes de las instalaciones hidráulicas y de electricidad serían a la vista, facilitando así su instalación y mantenimiento.

El proyecto piloto fue sometido a cálculos cuantitativos, abarcando 18 sistemas, cada cual calculado por un alumno, mediante supervisión de las profesoras, incluyéndose:

- Demoliciones

- Terraplenes

- Fundaciones

- Estructura

- Contrapiso

- Impermeabilización

- Cierres

- Puertas y ventanas

- Aislamiento

- Cubiertas

- Forros

- Pisos

- Equipamientos especiales

- Entresuelo

- Porcelanas sanitarias y accesorios

- Electricidad

- Hidráulica

- Cierres externos 
El proyecto y los cuantitativos fueron presentados en reunión entre el equipo de alumnos y profesoras tutora y coordinadora, el representante de la RECOOPERA, representantes del Ayuntamiento del Municipio y de la empresa patrocinadora. El proyecto fue aceptado con elogios y la empresa patrocinadora presentó los costos del presupuesto, siendo aprobados. Al momento de la elaboración de este artículo, se espera la aprobación final de las piezas gráficas por el Ayuntamiento y la autorización para el inicio de las obras de construcción por parte de la empresa patrocinadora. Se pretende la continuidad de la participación presentando el proyecto preliminar al conjunto de la cooperativa mediante láminas, maqueta y sistema constructivo para adaptaciones y desarrollo final.

\begin{tabular}{|l|}
\hline \multicolumn{1}{|c|}{ ESPECIFICACIÓN } \\
\hline 1. ZONA DE OBRAS \\
\hline 2. ESTRUCTURAS DE HORMIGÓN \\
\hline 3. ESTRUCTURAS METÁLICAS \\
\hline 4. FORROS \\
\hline 5. CIERRES \\
\hline 6. VENTANAS \\
\hline 7. REVESTIMIENTOS Y PINTURAS \\
\hline 8. PISOS \\
\hline 9. BANCADAS \\
\hline 10. INSTALACIÓNES HIDRÁULICAS \\
\hline 11. INSTALACIÓNES ELÉCTRICAS \\
\hline TOTAL \\
\hline MANO DE OBRA \\
\hline MATERIALES \\
\hline
\end{tabular}
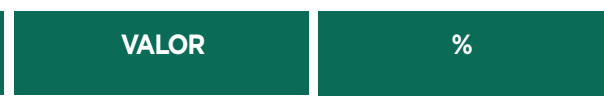

\begin{tabular}{|c|}
\hline$R \$ 129.552,00$ \\
\hline$R \$ 285.854,41$ \\
\hline$R \$ 518.991,02$ \\
\hline
\end{tabular}

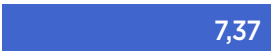

16,26

\begin{tabular}{|r|}
\hline$R \$ 30.181,50$ \\
\hline$R \$ 211.496,89$ \\
\hline
\end{tabular}

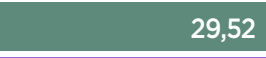

$\mathrm{R} \$ 211.496,89$

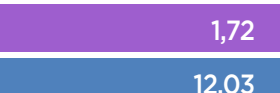

\begin{tabular}{|c|}
\hline$R \$ 83.642,45$ \\
\hline$R \$ 26.863,19$ \\
\hline
\end{tabular}

$R \$ 208.181,25$

$\mathrm{R} \$ 4.646,40$

$R \$ 105.991,00$

R\$ $152.543,28$

\begin{tabular}{|r|}
\hline 12,03 \\
\hline 4,76 \\
\hline 1,53 \\
\hline
\end{tabular}

\begin{tabular}{|r|}
\hline$R \$ 4.646,40$ \\
\hline$R \$ 105.991,00$ \\
\hline$R \$ 152.543,28$ \\
\hline$R \$ 1.757 .943,39$ \\
\hline
\end{tabular}

\begin{tabular}{|r|}
\hline 11,84 \\
\hline 0,26 \\
\hline 6,03 \\
\hline 8,68 \\
\hline $\mathbf{1 0 0 , 0 0}$ \\
\hline 41,76 \\
\hline $\mathbf{5 8 , 2 4}$ \\
\hline
\end{tabular}

\section{MANO DE OBRA $\times$ MATERIALES}

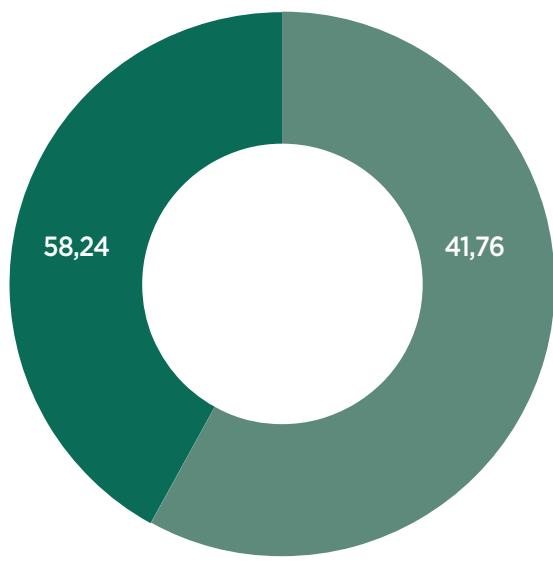

MANO DE OBRA
MATERIALES
TABLA DE GASTOS

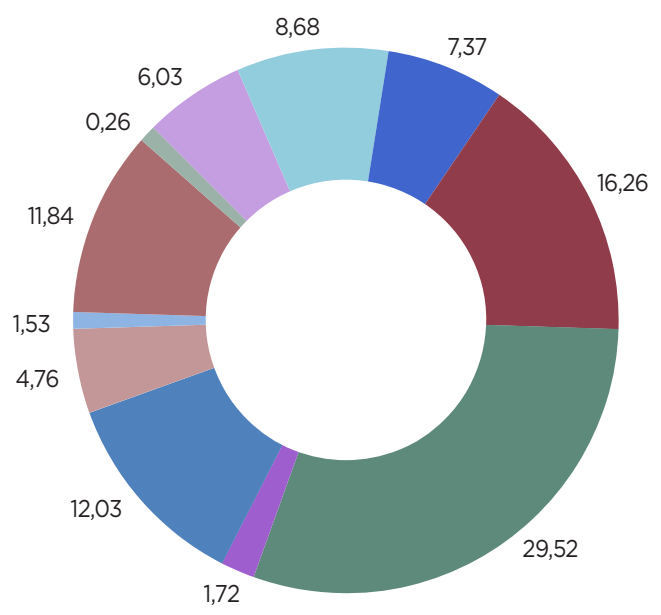

1. ZONA DE OBRAS

2. ESTRUCTURAS DE HORMIGÓN

3. ESTRUCTURAS METÁLICAS

4. FORROS

5. CIERRES

6. VENTANAS

7. REVESTIMIENTO Y PINTURAS

8. PISOS

9. BANCADAS

10. INSTALACIÓNES HIDRÁULICAS

11. INSTALACIÓNES ELÉCTRICAS

Figuras 8, 9 y 10. Proporción de costes (fuente: Equipo del PET). 


\section{CONCLUSIÓN.}

En cuanto al proyecto, la expectativa es que presente soluciones para mejorar las condiciones de trabajo y racionalidad productiva de las cooperativas. Se buscó reflexionar sobre el carácter y tipología de la arquitectura ligada a los usos productivos e industriales contemporáneos, sistematizada en el sentido de ofrecer una solución replicable, cuya ejemplaridad exprese, como valor funcional, estructural, constructivo y simbólico, la importancia de la actividad desde un punto de vista comunitario y ambiental.

Se espera que las soluciones de arreglo espacial y productivo puedan impactar de forma positiva a los miembros de la cooperativa involucrados, a la sociedad y al medio ambiente. Los propósitos de los procesos del trabajo académico y sus productos finales involucran la disposición de proponer un incremento real en la visibilidad de esta cooperativa, así como de otras, en su grado de reconocimiento por parte de la ciudadanía en el tejido social, como socialmente necesarias y realizadoras de un trabajo útil, innovador y respetable. Se busca reflexionar sobre el potencial de convenios como este en la elaboración de prácticas académicas que contemplen las diversas instancias del urbanismo y de la arquitectura a escala municipal.
La propuesta intentó, en los inicios de la realización del convenio, métodos y actividades, apuntar a caminos de enseñanza, investigación y extensión en conjunto que intensificaran el proceso de aprendizaje académico. Asimismo, se buscó un intercambio de conocimiento, desarrollo de habilidades y constitución de productos realizables, correspondientes a las necesidades y expectativas demandadas por la comunidad, sensibles y solidarios en la búsqueda de caminos compartidos, en el sentido de superar las condiciones de fragilidad socioespacial y ambiental que caracterizan a las poblaciones metropolitanas brasileñas y latinoamericanas.

\section{REFERENCIAS}

Ferreira, A.. Martins, J. y Freitas, R. 2007. "A experiência do Programa de Educação Tutorial." En Martins, I.

y Ketzer, S. (Org.), PET - Programa de Educação Tutorial: Estratégia para o desenvolvimento da graduaçāo Brasilia: MEC.

Silva, S. 2017. A organização coletiva de catadores de material reciclável no Brasil: Dilemas e potencialidades sob a ótica da economia solidária. Brasilia/ Rio de Janeiro: IPEA.

Disponible en: http://www.ipea.gov.br/portal/images/stories/PDFs/TDs/td_2268.pdf

Singer, P., 2008. "Economia solidária." Estudos Avançados, 22 (62): 289-314. 